\title{
Blue light negatively regulates the sexual filamentation via the Cwc1 and Cwc2 proteins in Cryptococcus neoformans
}

\author{
Ying-Ku Lu, Kai-Hui Sun and Wei-Chiang Shen* \\ Department of Plant Pathology and Microbiology, National \\ Taiwan University, 106 Taipei, Taiwan.
}

\section{Summary}

Cryptococcus neoformans is a heterothallic basidiomycetous yeast that primarily infects immunocompromised individuals. Dikaryotic hyphae resulting from the fusion of the MATa and MAT $\alpha$ mating type strains represent the filamentous stage in the sexual life cycle of $\boldsymbol{C}$. neoformans. In this study we demonstrate that the production of dikaryotic filaments is inhibited by blue light. To study blue light photoresponse in $C$. neoformans, we have identified and characterized two genes, $C W C 1$ and $C W C 2$, which are homologous to Neurospora crassa wc-1 and wc-2 genes. Conserved domain analyses indicate that the functions of Cwc1 and Cwc2 proteins may be evolutionally conserved. To dissect their roles in the light response, the $C W C 1$ gene deletion mutants are created in both mating type strains. Mating filamentation in the bilateral cross of cwc1 MATa and MAT $\alpha$ strains is not sensitive to light. The results indicate that Cwc1 may be an essential regulator of light responses in $C$. neoformans. Furthermore, overexpression of the CWC1 or CWC2 gene requires light activation to inhibit sexual filamentation, suggesting both genes may function together in the early step of blue light signalling. Taken together, our findings illustrate blue light negatively regulates the sexual filamentation via the Cwc1 and Cwc2 proteins in C. neoformans.

\section{Introduction}

All organisms are capable of sensing environmental fluctuations and make appropriate physiological adjustments via complex mechanisms. The capabilities to perceive light and adapt to its changes have been developed in organisms across different kingdoms. The processes of

Accepted 22 December, 2004. *For correspondence. E-mail wcshen@ntu.edu.tw; Tel. (+886) 223630231 ext. 2733; Fax (+886) 223636490 .

(C) 2005 Blackwell Publishing Ltd blue light perception, signal transduction and related responses have been extensively studied in bacteria, algae, plants and fungi. The classical genetic system $\mathrm{Neu}$ rospora crassa has been the leading model for studying the blue light photobiology in filamentous fungi (Linden et al., 1997; Liu et al., 2003).

Neurospora crassa can sense light in the ultraviolet and blue regions of the radiant energy spectrum, and in turn triggers diverse developmental, morphological and physiological responses (Degli-Innocenti and Russo, 1984). Blue light stimulates the formation of asexual sporulation structures and influences the number of conidial production (Lauter et al., 1997). Blue light also regulates the development of protoperithecia and the number of perithecia formed, and the perithecial beaks display positive phototropism towards blue light (Harding and Melles, 1983; Linden et al., 1997). Additionally, blue light induces the mycelial production of carotenoid pigment and acts as a signal to entrain the circadian rhythm (Sargent and Briggs, 1967; Harding and Turner, 1981).

Two $N$. crassa light-insensitive mutants, white collar-1 (wc-1) and white collar-2 (wc-2), were identified through extensive mutagenesis screens (Sargent and Briggs, 1967). wc-1 and wc-2 mutants exhibit pleiotropic phenotypes, and all the blue light-induced phenomena are abolished in the wc-1 or wc-2 null mutants (Linden et al., 1997). In the white collar mutant backgrounds, known genes under the control of blue light all lose their lightinducible transcriptional activation (Nelson et al., 1989; Schmidhauser et al., 1990; Lauter and Russo, 1991; Arpaia et al., 1993; 1995; Dunlap, 1996), suggesting that the gene products of $w c-1$ and $w c-2$ play pivotal roles in the regulation of blue light responses in $N$. crassa.

Neurospora crassa $w c-1$ and $w c-2$ genes have been cloned and characterized (Carattoli et al., 1995; Ballario et al., 1996; Linden and Macino, 1997). Analyses of the deduced amino acid sequences of these proteins

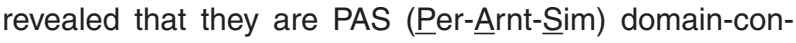
taining transcriptional factors with GATA type zinc finger DNA-binding domains. Subsequent studies demonstrated that WC-1 and WC-2 proteins form heterodimeric complex via the PAS domains to activate the transcription of downstream light-regulated genes (Ballario et al., 1998; Cheng et al., 2002). A specialized class of PAS domains known 
as LOV (for Light-Oxygen-oltage) was identified in WC1 protein. The LOV domain of WC-1 was found to be similar to the chromophore-binding LOV domains of phototropins, which are blue light photoreceptors in plants (Huala et al., 1997; Crosson and Moffat, 2001). WC-1 protein was later demonstrated to be the blue light photoreceptor in N. crassa (Froehlich et al., 2002; He et al., 2002) and a flavin species, flavin adenine dinucleotide (FAD), was shown to be the chromophore associated with WC-1 through binding to the LOV domain. This is the first demonstration of fungal blue light photoreceptor.

Cryptococcus neoformans is an opportunistic pathogenic yeast that infects both immunocompromised and immunocompetent individuals (Casadevall and Perfect, 1998). This fungus has recently become a model system of fungal pathogenesis, physiology and differentiation because of increasing clinical importance and well-developed molecular and genetic tools (Lengeler et al., 2000; Hull and Heitman, 2002).

Cryptococcus neoformans is a heterothallic basidiomycete and grows vegetatively as a yeast. The sexual and asexual life cycles have been well described (KwonChung, 1975; 1976; Alspaugh et al., 2000). Under nitrogen limitation conditions, strains of opposite mating types, MATa and MAT $\alpha$, produce conjugation tubes and fuse to form dikaryotic filaments with fused clamp connections. The tips of the filaments further differentiate to form the basidia, where nuclear fusion and meiosis occur, and four chains of sessile basidiospores are finally produced. An asexual sporulation process called haploid fruiting or haploid filamentation was originally described in MAT $\alpha$ strains and this phenomenon has recently been observed also in MATa cells (Wickes et al., 1996; Tscharke et al., 2003). This process primarily occurs under nitrogen starvation and severe desiccation conditions and has been suggested as a way to relocate the $C$. neoformans cells to find their mating partners (Wickes et al., 1996).

Studies of the $C$. neoformans genes involved in the mating process revealed that the production of dikaryotic filaments during the mating process is influenced by light. No known photoresponse has been previously described in $C$. neoformans. To further characterize this phenomenon, we examined the effectiveness of different wavelength of light and the molecular mechanism of photoresponse in $C$. neoformans. Here we report the identification and characterization of the $N$. crassa wc-1 and wc2 homologues in C. neoformans. We found that Cwc1 and Cwc2 proteins are essential during signalling of blue lightregulated mating process in $C$. neoformans and may function in a way similar to $N$. crassa WC-1 and WC-2 proteins. Our results suggest that cellular components involved in the perception and control of light responses may be evolutionally conserved in this distantly related human fungal pathogen.

\section{Results}

Blue light inhibits the production of dikaryotic mating filaments in C. neoformans

Cryptococcus neoformans grows vegetatively as a yeast under normal growth conditions, and filamentous hyphae develop predominantly in the process of sexual differentiation. In an effort to characterize genes involved in the mating process, we found that the formation of dikaryotic filaments was inhibited by light (Shen et al., 2002). To confirm this observation, we utilized the serotype D MAT $\alpha$ and MATa wild-type cells and compared the mating reactions under constant white light and dark conditions. We found that white light inhibited the development of the dikaryotic mating filaments, and the filaments developed under such condition were fewer and shorter (Fig. 1A). In contrast, abundant and longer mating filaments were developed in darkness after the same incubation period (Fig. 1A). Although the production of sexual filaments is apparently affected by light, the mating differentiation is not blocked by light. Filamentation can be observed after prolonged incubation, and the sexual progeny can still be obtained. In addition, light inhibition of dikaryotic filaments during the mating process is not restricted to V8 mating medium, and we have seen this phenomenon on other mating supporting media including synthetic low-ammonium dextrose (SLAD) and FA media (data not shown). Furthermore, the light sensitivity is not restricted to the serotype $\mathrm{D} C$. neoformans strains. The mating filaments produced from the cross of the serotype A MAT $\alpha$ wild-type strain H99 and serotype D MATa wild-type strain JEC20 are also inhibited by light (Fig. 1B).

To determine the effectiveness of wavelength for triggering $C$. neoformans photoresponses, we set up illumination systems within three different wavelength ranges in the visible light spectrum including blue, red and far-red light (Peter et al., 1998). The results indicate that blue light strongly inhibits the production and growth of dikaryotic filaments (Fig. 1A). On the other hand, the production of filaments is unaffected by red light and far-red light and filamentation under these two conditions is comparable to the sample observed in darkness (Fig. 1A). Taken together, our findings demonstrate that $C$. neoformans can sense light within the blue region of the visible light spectrum and blue light inhibits the production of dikaryotic mating filaments.

Identification and characterization of the C. neoformans CWC1 and CWC2 genes

We demonstrate that $C$. neoformans is able to perceive blue light and regulates the filamentous morphogenesis associated with the sexual mating process. In fungi, molecular mechanisms of blue light perception and regu- 
A

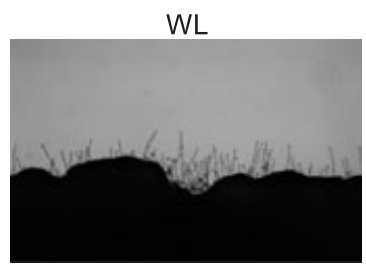

$B L$
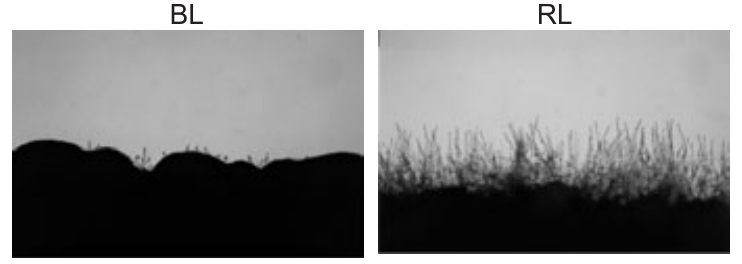

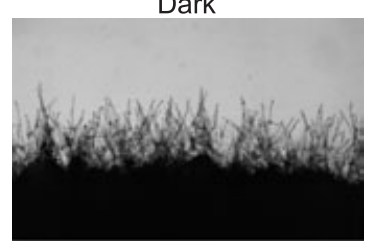

FRL

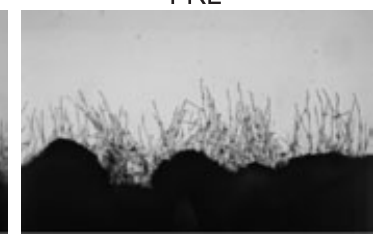

B
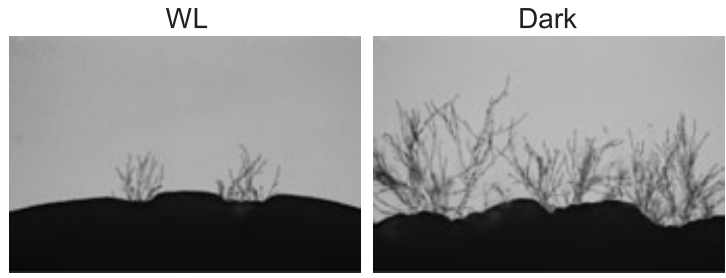

Fig. 1. Blue light inhibits the production of dikaryotic mating filaments in C. neoformans. A. Congenic serotype D wild-type MATa (JEC 20) and $M A T \alpha$ (JEC 21) strains were mixed and incubated on V8 mating agar for 2 days at $22^{\circ} \mathrm{C}$ with different illumination conditions. WL: the V8 mating plate was incubated under white light condition $\left(84 \mu \mathrm{mol} \mathrm{m}^{-2} \mathrm{~s}^{-1}\right)$; Dark: the V8 mating plate was incubated in the dark; $B L, R L$, FRL: the V8 mating plates were incubated under blue light $\left(11 \mu \mathrm{mol} \mathrm{m}^{-2} \mathrm{~s}^{-1}\right)$, red light $\left(27 \mu \mathrm{mol} \mathrm{m} \mathrm{m}^{-2} \mathrm{~s}^{-1}\right)$, far-red light $\left(33 \mu \mathrm{mol} \mathrm{m}{ }^{-2} \mathrm{~s}^{-1}\right)$ conditions respectively. The edges of mating spots were photographed at $100 x$ magnification.

B. The serotype A MAT $\alpha$ wild-type strain H99 and serotype D MATa wild-type strain JEC20 were mixed and incubated on V8 mating agar for 4 days at $22^{\circ} \mathrm{C}$ under white light (WL, $7 \mu \mathrm{mol} \mathrm{m}{ }^{-2} \mathrm{~s}^{-1}$ ) or constant darkness conditions. lation have been studied in great details in N. crassa, and WC-1 and WC-2 proteins have been shown to play the central roles in the photoresponses of $N$. crassa. To dissect the molecular mechanisms of light response in $C$. neoformans, we used $N$. crassa WC-1 and WC-2 amino acid sequences to conduct BLAST search analyses and found candidate homologues in the Standford C. neoformans genome database. BLAST search with the retrieved sequences in GenBank further confirmed their homologies with $N$. crassa WC-1 and WC-2, and we therefore named the homologues CWC1 (Cryptococcus WC-1 homologue) and CWC2 (C ryptococcus WC-2 homologue). Oligonucleotide primers were designed to amplify the corresponding CWC1 and CWC2 fragments and the amplified products were used as probes to identify the respective genomic clones. Reverse transcription polymerase chain reaction (RT-PCR) was conducted to obtain the CWC1 cDNA fragments. Sequencing analyses of CWC1 genomic clone and RT-PCR products revealed a predicted coding region of $3591 \mathrm{bp}$ with three introns, encoding for a protein of 1141 amino acid residues. Analyses of CWC2 genomic sequence indicated a $1439 \mathrm{bp}$ coding region encoding a 392-amino-acid protein with four introns.

Conserved domain and BLAST analyses revealed several important features in the Cwc1 and Cwc2 polypeptides. The predicted structural organizations of $C$. neoformans Cwc1 and Cwc2 proteins are shown and compared with the functional domains of $N$. crassa WC-1 and WC-2 (Fig. 2). A putative LOV domain, two putative PAS domains, and a putative nuclear localization sequences are identified in the $C$. neoformans Cwc1 protein, whereas the Cwc2 protein includes a putative PAS domain and GATA type zinc finger DNA-binding domain.

Overall, the C. neoformans Cwc1 and N. crassa WC-1 proteins share $18 \%$ identity and $28 \%$ similarity. Sequence conservation is more significant among the predicted functional domains. The sequences of the LOV domains of $C$. neoformans Cwc1 and N. crassa WC-1 proteins are about $57 \%$ identity and $76 \%$ similarity, and the first and second PAS domains found in both proteins share $40 \%$ sequence identity and 55\% similarity, and $57 \%$ identity and $76 \%$ similarity respectively.

On the other hand, the sequences of $C$. neoformans Cwc2 and N. crassa WC-2 proteins share $16 \%$ identity

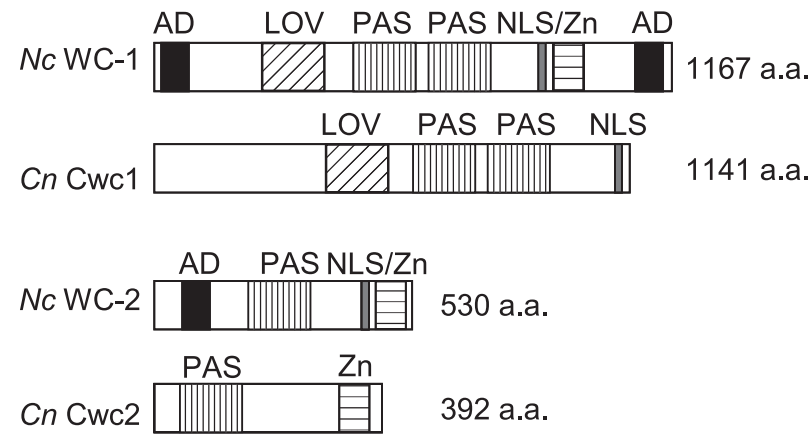

Fig. 2. Schematic representation showing the domains organization of $C$. neoformans CwC1 and N. crassa WC-1, and C. neoformans Cwc2 and N. crassa WC-2. AD, the putative activation domains; LOV, the Light-Oxygen-Voltage domains; PAS, the Per-Arnt-Sim domains; NLS, the putative nuclear localization signals; $Z n$, the zinc finger DNA-binding domains; a.a., amino acids. 
and $27 \%$ similarity. Greater sequence conservation is found in the zinc finger DNA-binding domains, and the PAS domains among the two proteins only share $20 \%$ sequence identity and $41 \%$ similarity.

\section{Disruption of the C. neoformans CWC1 gene}

The function of the CWC1 gene in C. neoformans was further analysed by gene disruption. The MAT $\alpha$ wild-type copy of the CWC1 gene was replaced with the cwC1::URA5 disruption allele by homologous recombination (Fig. 3A). Mutant strains were screened by PCR and verified by Southern blot analysis. The hybridization results confirmed the gene replacement by the presence of $5.2 \mathrm{~kb} \mathrm{EcoRl}$ hybridization signal due to the loss of $E C O R I$ restriction site in the deleted region of $C W C 1$ gene and the absence of $2.9 \mathrm{~kb}$ wild-type fragment (Fig. 3B).

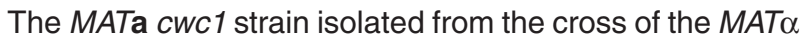
cwC1 strain and JEC34 (MATa ura5) was also confirmed by Southern hybridization analysis (Fig. 3B). Furthermore, wild-type fragment of $C W C 1$ gene was reintroduced into

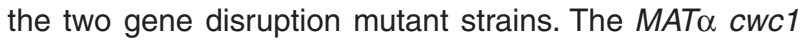
and MATa $c W C 1$ strains with the reintroduction of the wildtype fragment of CWC1 gene were also obtained and verified (Fig. 3B).

\section{Deletion of the CWC1 gene results in loss of light} inhibition of filamentation during the mating process

The phenotypes of the MAT $\alpha$ CWC1 and MATa $c W c 1$ mutant strains were examined and the formation of dikaryotic filaments was compared under constant light and dark conditions. Mating between the wild-type MATa (JEC20)

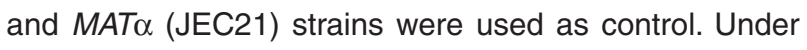
constant darkness abundant sexual filaments were observed (Fig. 4A). In contrast, the inhibition of filamentation by light was observed on V8 mating medium at $22^{\circ} \mathrm{C}$ after $24 \mathrm{~h}$ incubation, and only a few filaments were observed at the edges of the mating strains in white light condition (Fig. 4D). When the MATa $c w c 1$ or MAT $\alpha$ cwc1 strain was co-incubated with the opposite MAT $\alpha$ or MATa mating type wild-type tester strains, more filaments were formed in light condition as compared with the cross between the MATa and MAT $\alpha$ wild-type strains (Fig. 4E and F); however, the filaments were not as many as those formed in darkness (Fig. 4B and C). Interestingly, more dramatic production of mating filaments was observed in the bilateral cross of the MAT $\alpha$ CWC1 and MATa $c w c 1$ strains under constant light illumination (Fig. $4 \mathrm{~J}$ ), and the amount of the mating filaments under light was comparable to that formed in the dark (Fig. 4G). Finally, unilateral or bilateral cross of MAT $\mathrm{CWC1}$ or MATa CWC1 reconstitution strains under light and dark conditions was conducted, and the extent of filamentation in these crosses was all comparable to the extent of filamentation in the wild-type strain background (Fig. $4 \mathrm{H}, \mathrm{I}, \mathrm{K}$ and L; data not shown). Taken together, our results demonstrate that deletion of the CWC1 gene results in loss of light sensitivity, and the sexual filamentation is no longer inhibited by light.

\section{The CWC1 and CWC2 genes are expressed at a} very low level

To understand the regulation of CWC1 and CWC2 in response to light, the steady-state level of mRNA expression under different illuminaton conditions was examined. Total RNA was extracted from the MAT $\alpha$ wild-type strain grown under conditions with constant light, constant darkness and constant darkness with 20 min or $2 \mathrm{~h}$ light induction before harvesting the cells during the $24 \mathrm{~h}$ incubation period in the yeast-peptone-dextrose (YPD) liquid medium. Twenty micrograms of total RNA from each sample were subjected to Northern hybridization analysis. However, under all conditions tested we failed to detect the CWC1 and CWC2 RNA transcripts (Fig. 5A). The results suggest that both of the $C$. neoformans CWC1 and CWC2 genes are expressed at a very low level.
A
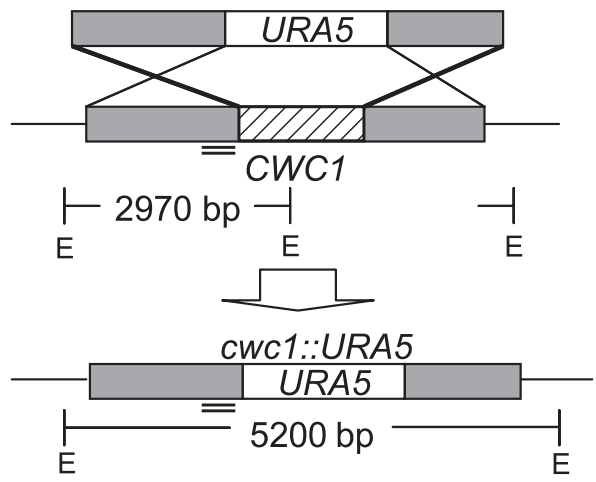

B

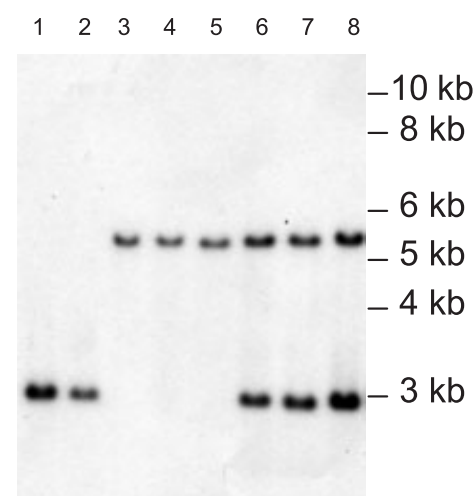

Fig. 3. Disruption of the CWC1 gene.

A. The cWc1::URA5 deletion allele was created by replacing part of the $C W C 1$ coding region with the URA5 selectable marker. The hybridization probe for Southern blot analysis is indicated by double line. E, EcoRI

B. Genomic DNA from the MAT $\alpha$ wild-type (lane 1, JEC21), MATa wild-type (lane 2, JEC20), cwC1 MATo mutant strains (lane 3 and 4, YKC7 and YKC12), cwc1 MATa mutant strain (lane 5, YKC29), cWC1 + CWC1 MAT $\alpha$ reconstituted strains (lanes 6 and 7, YKC32 and YKC33) and cWC1 + CWC1 MATa reconstituted strain (lane 8, YKC34) was digested with EcoRI and subjected to Southern hybridization analysis using the CWC1 probe indicated in (A). Size standards are indicated by kilobases (kb). 

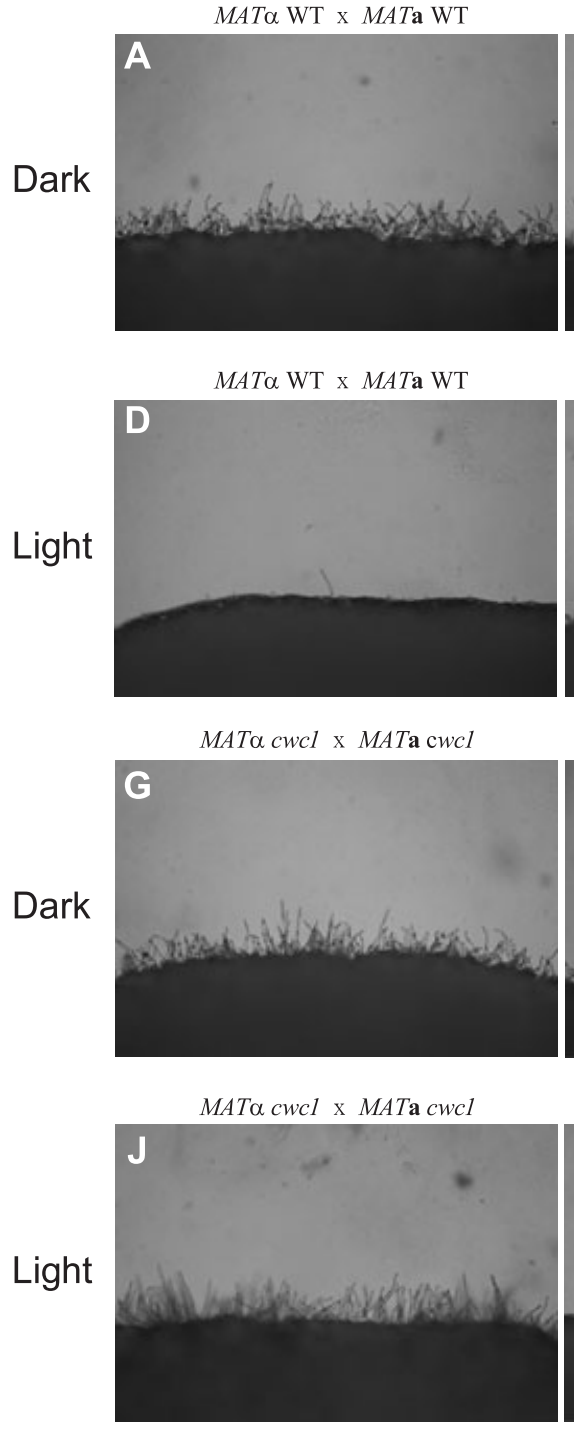
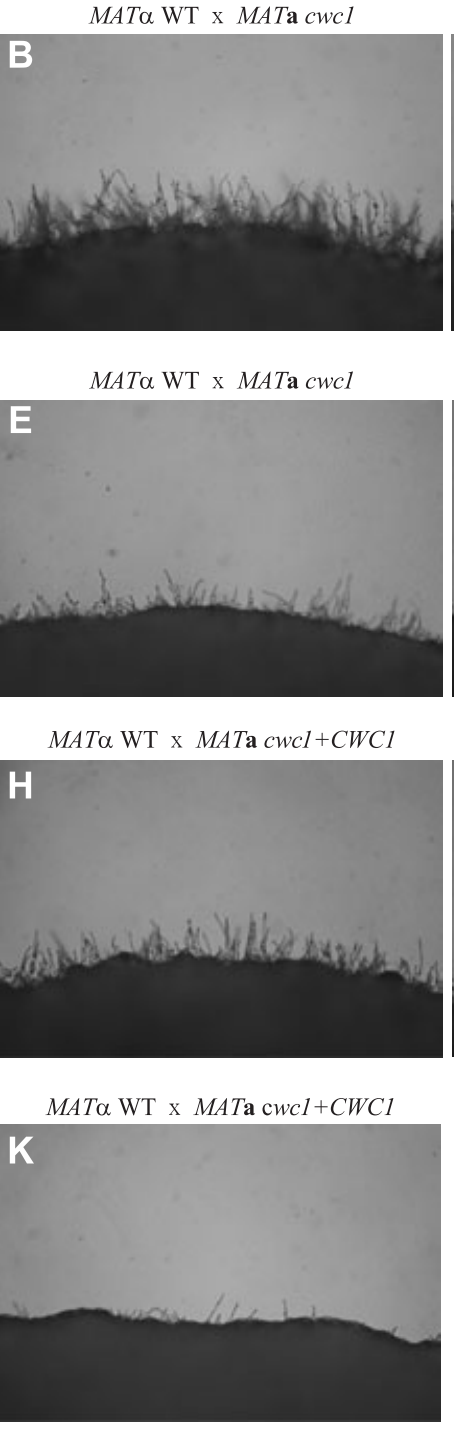

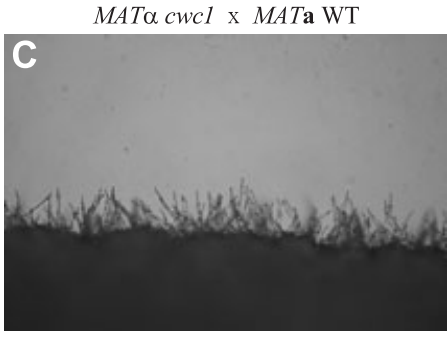

MAT $\alpha$ cwcl x MATa WT

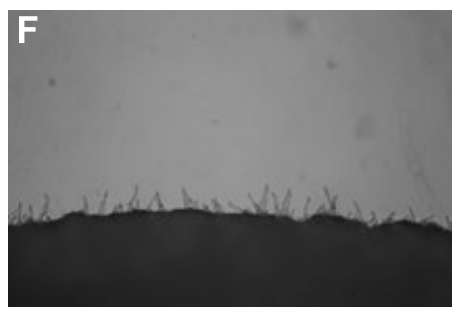

$M A T \alpha \mathrm{c} w c 1+C W C 1 \mathrm{X} M A T \mathbf{a} c w c 1+C W C 1$

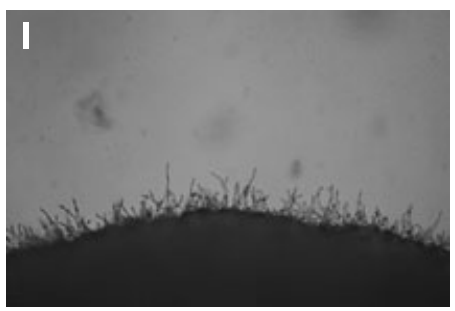

$M A T \alpha \mathrm{c} w c 1+C W C 1 \times \mathrm{X} M A T \mathbf{a} c w c 1+C W C l$

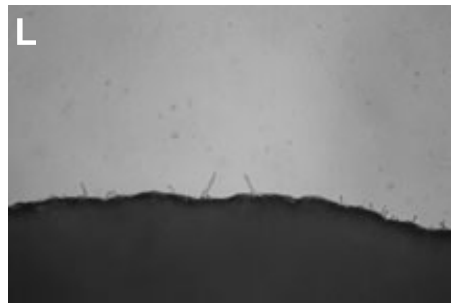

Fig. 4. Deletion of the CWC1 gene results in loss of light inhibition of filamentation in the mating process. The MAT $\alpha$ wild-type (JEC21), $C W C 1$ MAT $\alpha$ mutant strain (YKC7), and cwc1 + CWC1 MAT $\alpha$ reconstituted strain (YKC32) were individually crossed with the MATa wild-type (JEC20) or cWC1 MATa mutant strain (YKC29) or cWC1 + CWC1 MATa reconstituted strain (YKC34) as indicated under constant darkness and white light $\left(7 \mu \mathrm{mol} \mathrm{m} \mathrm{m}^{-2} \mathrm{~s}^{-1}\right)$ conditions. Mating was conducted on V8 agar at $22^{\circ} \mathrm{C}$, and photos were taken after $24 \mathrm{~h}$ incubation at $100 \times$ magnification.

To further reveal the possible pattern of their regulation, we conducted the real-time PCR detection approach. The same amount of RNA from each sample used for Northern blot analysis was reverse transcribed with oligo-dT primer. Two sets of primer pairs optimized for real-time PCR analysis were designed in the CWC1 and CWC2 coding regions. To normalize the RNA sample, the constitutively expressed C. neoformans glyceraldehyde 3-phosphate dehydrogenase gene GPD1 was used as control. The standard curves for all the three genes were experimentally determined and the dilution range for the analysis of each gene was also optimized. The resulting PCR amplification curves of CWC1 and CWC2 indicated that the steady states of $C W C 1$ and $C W C 2$ transcripts are very low under the conditions examined. However, relative changes of the CWC1 and CWC2 mRNA levels under different illumination conditions can be detected. The results of real-time PCR analyses are shown in Fig. 5B. C. neoformans CWC1 appears to be expressed at a constitutive level. On the other hand, slightly induction of the CWC2 message was detected in the cells grown in darkness with 20 min light induction, and no significant difference is observed under other conditions. Taken together, our real-time PCR analyses indicate that both the CWC1 and $C W C 2$ genes are expressed at very low levels. The CWC1 gene is constitutively expressed, and the steadystate level of CWC2 mRNA may possibly be regulated by light in C. neoformans. 
A

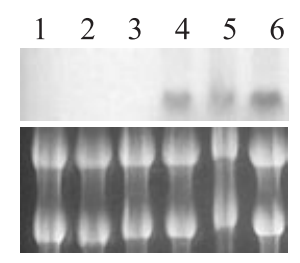

C
B

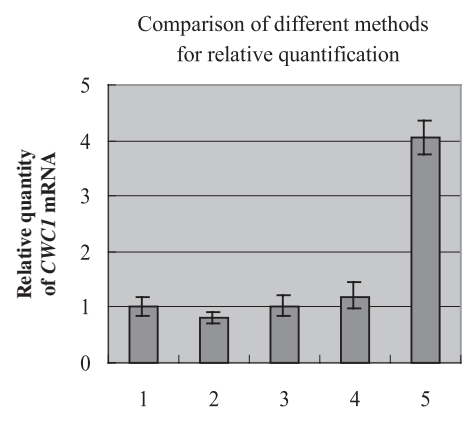

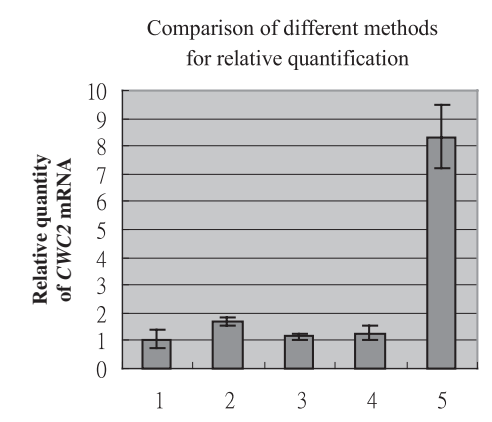

mparison of different methods for relative quantification

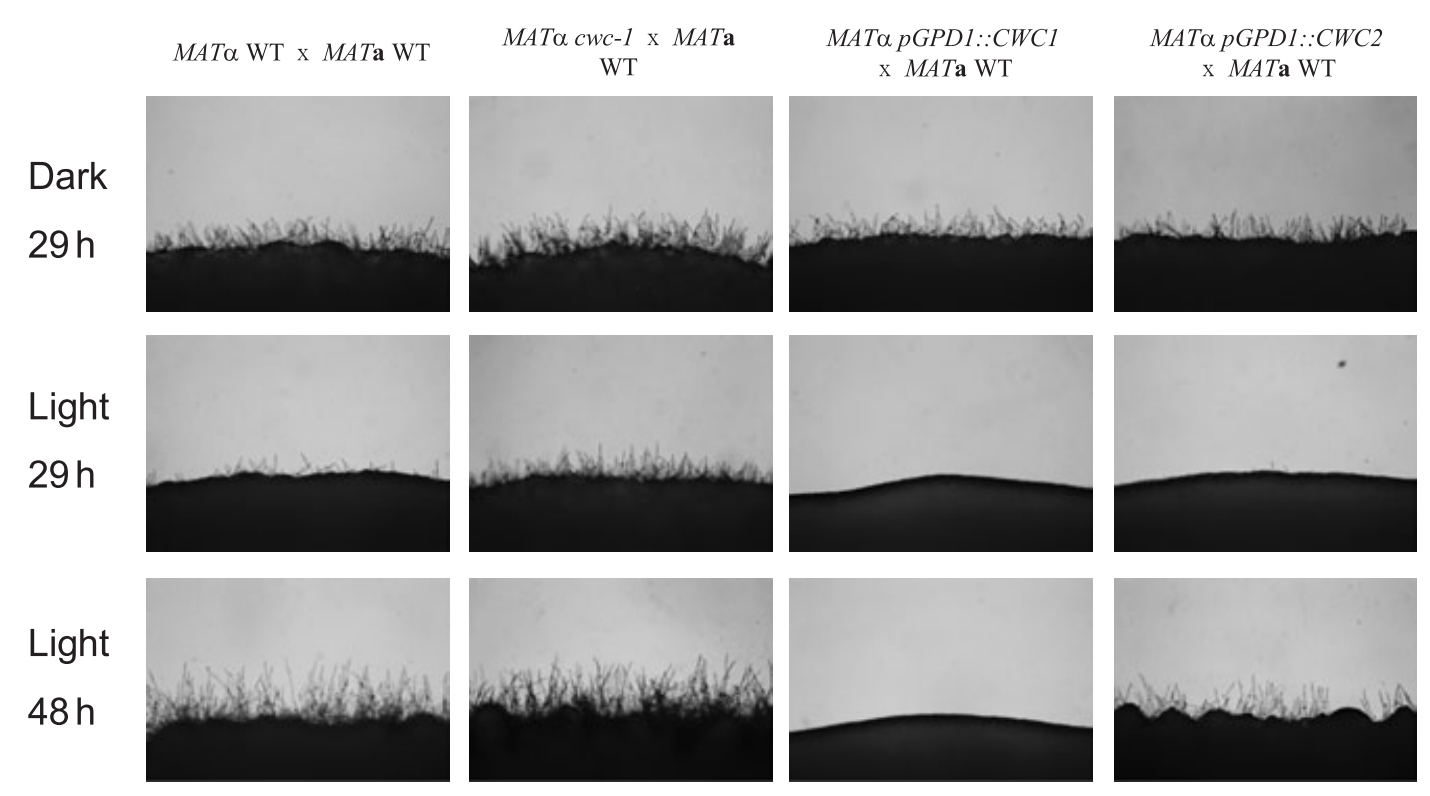

Fig. 5. Inhibition of the sexual filamentation by $C W C 1$ and $C W C 2$ overexpression requires light activation.

A. Northern analysis of $P_{G P D 1}-C W C 1$ overexpression strains. Total RNA extracted from the MAT $\alpha$ wild-type (lane 1, JEC21), cWC1 MAT $\alpha$ mutant strain (lane 2, YKC7), cWC1 + CWC1 MAT $\alpha$ reconstituted mutant strain (lane 3, YKC32) and $P_{G P D 1}$-CWC1 overexpression strains (lane 4, YKC38; lane 5, YKC39; lane 6, YKC40) was subjected to Northern blot analysis. Hybridization probe used was as indicated in Southern analysis. RNA loading is shown by the ethidium-bromide-stained gel.

B. Relative real-time PCR quantification of CWC1 (left) and CWC2 (right) mRNA in the MAT $\alpha$ wild-type strain (JEC21), CWC1 (YKC38) and CWC2 (KHC6) overexpression strains. RNA samples of the MAT $\alpha$ wild-type strain were extracted from growth conditions in the constant darkness (1), and constant darkness with $20 \mathrm{~min}(2)$ or $2 \mathrm{~h} \mathrm{(3)} \mathrm{light} \mathrm{induction} \mathrm{before} \mathrm{harvesting} \mathrm{the} \mathrm{cells,} \mathrm{and} \mathrm{constant} \mathrm{light} \mathrm{(4)} \mathrm{conditions} \mathrm{during} \mathrm{the} 24 \mathrm{~h}$ incubation period in the YPD liquid medium. The results of RNA extracted from the $P_{G P D 1}-C W C 1$ (YKC38) and $P_{G P D 1}-C W C 2$ (KHC6) overexpression strains grown in the YPD liquid medium are shown in column (5). The results were normalized by the GPD1 mRNA. $n=3$ for all groups.

C. The CWC1 (YKC38) and CWC2 (KHC6) overexpression strains were crossed with the MATa wild-type (JEC20) under dark and light conditions. The MAT $\alpha$ wild-type (JEC21) and the cwC1 MAT $\alpha$ mutant strain (YKC7) were co-inoculated as controls for the mating reaction. Mating was conducted on V8 agar at $22^{\circ} \mathrm{C}$ under constant dark and white light $\left(7 \mu \mathrm{mol} \mathrm{m}^{-2} \mathrm{~s}^{-1}\right)$ conditions, and photos were taken after 29 and $48 \mathrm{~h}$ incubation as indicated at $100 \times$ magnification.

\section{Inhibition of the filamentation by overexpression of CWC1 and CWC2 requires light activation}

To further reveal the molecular mechanism of CWC1 function, the promoter of $C$. neoformans GPD1 gene was used to examine the effect of $C W C 1$ overexpression. The fusion construct of candidate $C$. neoformans $C W C 1$ overexpression strains was verified by PCR. The expression of CWC1 transcripts in the overexpression strains was con- firmed by Northern hybridization analysis (Fig. 5A). Realtime PCR analyses revealed at least fourfold induction was observed among the CWC1 overexpression strains (Fig. 5B and data not shown). The confirmed CWC1 overexpressers were subjected to phenotypic characterization of dikaryotic filamentation. Interestingly, the presence of the CWC1 transcripts and proteins was not enough for such inhibition to occur under constant darkness condition. Inhibition of the dikaryotic filaments fully depended 
on light, and upon light induction formation of the dikaryotic mating filaments was completely repressed by $C W C 1$ overexpression even after $48 \mathrm{~h}$ prolonged incubation (Fig. 5C and data not shown).

Similar experiments were also conducted to examine the effect of CWC2 overexpression. The CWC2 overexpression strain was verified by PCR and subjected to realtime PCR analysis. An about eightfold elevation of the steady state of CWC2 mRNA was detected in the CWC2 overexpresser by real-time PCR analysis (Fig. 5B). Overexpression of the CWC2 gene also failed to inhibit dikaryotic filament formation in darkness. Upon light illumination, the production of sexual filaments was repressed to some extent but not completely and some filaments were observed after prolonged light illumination (Fig. 5C). Taken together, we conclude that the inhibition of the sexual filamentation process by light in $C$. neoformans is mediated via the Cwc1 and Cwc2 proteins, and light activation is required for proper execution of molecular functions of the Cwc1 and Cwc2 proteins.

\section{Discussion}

Light is an important environmental cue regulating diverse physiological and developmental processes. The effects of light on fungi have been well documented (Carlile, 1965; Tan, 1978). Most fungi respond to near UV and blue light, but for some fungi, including Aspergillus nidulans, biological responses to red and far-red light have been reported (Mooney and Yager, 1990; Griffith et al., 1994). In this study, we report the first photoresponse of $C$. neoformans and demonstrate that a robust filamentation process associated with sexual differentiation in C. neoformans is negatively regulated by light. Using several filters, we determined that $C$. neoformans can sense blue light but is not influenced by red or far-red light. The perception of blue light results in inhibition of dikaryotic filaments formation, and hyphae produced under blue light are much sparser and shorter compared with other illumination conditions (Fig. 1A). This inhibition phenomenon appears to be universal, and is not restricted to certain $C$. neoformans serotypes or media (Fig. 1). In fact, we found that diploid filamentation associated with certain $C$. neoformans diploid strains is also inhibited by light (Sia et al., 2000; data not shown).

Blue light photoresponses have been studied in great detail in $N$. crassa and interactions among the functional domains of two central regulatory proteins, WC-1 and WC2 , allow the light-induced processes to be delicately regulated. By reverse genetics approach, we identified the $N$. crassa wc-1 and wc-2 homologues, CWC1 and CWC2, in C. neoformans. Deletion of the CWC1 gene results in mutant strains insensitive to light. In the unilateral crosses between MAT $\alpha$ or MATa $c W c 1$ mutant and the opposite mating type wild-type strain, light inhibition still occurs to some extent due to the presence of one CWC1 wild-type copy (Fig. 4E and F). In the bilateral cross of the cwc1 $M A T \alpha$ and MATa cells, light inhibition of mating filamentation is abolished, and more dramatic production of mating filaments is observed under constant light condition and the extent is comparable to that observed in cross between the wild-type MAT $\alpha$ and MATa cells in darkness (Fig. 4J and A). These findings suggest that CWC1 is an important regulator of light response in $C$. neoformans and light-dependent inhibition of sexual filamentation is mediated by the level of Cwc1 inside the cells.

The perception of blue light in $N$. crassa is mediated by the photoreceptor WC-1 (Froehlich et al., 2002; He et al., 2002). N. crassa strains with the deleted or point-mutated LOV domain version of WC-1 protein lose light responses (Ballario et al., 1998; He et al., 2002). Conservation of 11 amino acid residues important for chromophore binding was identified in the LOV domain of $N$. crassa WC-1 (Christie et al., 1999). In C. neoformans Cwc1 protein, a putative LOV domain was identified (Fig. 2). Sequence alignment of Cwc1 LOV domain with LOV domains identified from other homologues found that these 11 amino acid residues are also conserved in Cwc1. The findings strongly suggest that the LOV domain of Cwc1 may share the conserved function and involve in chromophore binding.

In addition, alignment of LOV domains revealed a segment of 9-11 amino acid residues is inserted between $\alpha$ 'A and $\alpha \mathrm{C}$ loop in certain LOV domains containing homologues including $N$. crassa WC-1 (Crosson et al., 2003). This structure was suggested to possibly accommodate the larger, terminal adenine moiety of FAD, which has been demonstrated to be the flavin species associated with N. crassa WC-1 (Froehlich et al., 2002; He et al., 2002; Crosson et al., 2003). This 11-amino-acid segment is also present in $C$. neoformans Cwc1. Whether FAD is the chromophore binding to Cwc1 remains to be determined by fluorescence spectroscopic analysis.

To reveal the function of CWC2 gene in the photoresponses of $C$. neoformans, the cwC2::URA5 disruption allele was also created and transformed. Preliminary phenotypic characterization indicated $c w c 2$ mutants displayed similar phenotypes as $c w c 1$ mutants. The result suggests that Cwc2 may also function in the blue light response pathway in this basidiomycetous pathogen.

The results of overexpression experiments are intriguing. Strains overexpressing CWC1 gene display normal sexual filamentation in darkness as the wild-type MAT $\alpha$ cells. However, complete inhibition of mating filaments is observed upon light activation (Fig. 5C). This light-driven change phenotype further suggests that Cwc1 functions as a blue light photoreceptor in $C$. neoformans and the Cwc1 proteins must be converted to an active form by light 
to perform its function. The observation that overexpression of CWC2 dramatically but not completely inhibited the sexual filamentation upon light exposure is also interesting (Fig. 5C). Lack of conserved chromophore binding LOV domain in Cwc2 suggests Cwc2 itself is unlikely to function as a molecule for light perception. In N. crassa, the WC-1 and WC-2 proteins dimerize via the PAS domains (Ballario et al., 1998; Cheng et al., 2002), and WC-1 has also demonstrated to be the limiting factor in the complex (Cheng et al., 2001). The presence of PAS domain in Cwc2 suggests that it may interact with a less abundant light-activating component to properly execute its function.

From the presence of PAS domains in Cwc1 and Cwc2 and similar phenotypes observed in the CWC1 and CWC2 gene deletion or overexpression strains, we hypothesize that C. neoformans Cwc1 and Cwc2 possibly form the complex via the conserved protein dimerization PAS domains to activate downstream related genes. However, domain organization in $C$. neoformans Cwc proteins somewhat differs from that in N. crassa WC proteins. First, the zinc finger DNA-binding domain is not found in Cwc1, but present in Cwc2 in C. neoformans. In N. crassa, the zinc finger DNA-binding domains are present in both WC1 and WC-2 proteins. However, light induction requires only one intact zinc finger DNA-binding domain from either WC-1 or WC-2 (Cheng et al., 2003). Second, the nuclear localization sequence has only identified in Cwc1. In $N$. crassa, the nuclear localization sequences are also found in both WC-1 and WC-2 proteins. Localization study has demonstrated that WC-1 is localized exclusively in the nucleus, and WC-2 is found in both nuclear and cytoplasmic fractions (Schwerdtfeger and Linden, 2000). Further experiments will be required to address the subcellular distribution of Cwc proteins. Finally, no corresponding activation domain has been identified in $C$. neoformans Cwc1 and Cwc2 proteins. Further structural analysis of the Cwc proteins should help to define regions important for their regulation. Overall, sequence analyses revealed that even though the conserved photoresponse proteins are present in these two evolutionarily distinct organisms, and some underlying molecular execution of Cwc1 and Cwc2 proteins may be different from WC-1 and WC-2 proteins of $N$. crassa. How Cwc1 and Cwc2 proteins function together in $C$. neoformans remains to be answered.

The expression of CWC1 and CWC2 genes was also investigated. The steady-state mRNA level of both genes was very low and failed to detect by Northern analysis (Fig. 5A). Using real-time PCR technique, we revealed that the CWC1 gene is constitutively expressed, and the expression of CWC2 gene is slightly upregulated in response to light and then goes down to the basal level after $2 \mathrm{~h}$ (Fig. 5B). Whether this upregulation is significant needs further examination. The expression levels of $w c-1$ and $w c-2$ genes in $N$. crassa appears to be higher compared with those of CWC1 and CWC2 in C. neoformans, and both $w c-1$ and $w c-2$ is transcriptionally induced upon 20 min light irradiation by Northern analysis (Ballario et al., 1996; Linden and Macino, 1997). Additionally, the expression of $w c-1$ is autoregulated and PAS domainmediated WC-1/WC-2 interaction is essential for maintaining the steady-state level of $N$. crassa $w c-1$ transcript (Ballario et al., 1996; Cheng et al., 2002). To further understand the regulation of CWC genes, we are currently examining the CWC2 expression in the $C W C 1$ mutant background to determine whether the light induction of CWC2 is dependent on the function of Cwc1.

To summarize, in this study negative regulation of sexual filamentation by blue light is demonstrated in $C$. neoformans. Two conserved proteins, Cwc1 and Cwc2, are identified and shown to be involved in the regulation of light response in this pathogenic fungus. However, the specific roles of these two proteins need further investigation. On the other hand, based on our current results we propose two models to describe the molecular control of photoresponses by the Cwc proteins in C. neoformans (Fig. 6). In both models, Cwc1 functions as a blue light photoreceptor to mediate light responses through complexing with Cwc2. The unidentified chromophore molecule, possibly FAD, is non-covalently associated with the Cwc1/Cwc2 complex via the LOV domain of Cwc1. Upon light induction, covalent modification occurs between the chromophore and LOV domain and results in the conformational change of the Cwc1/Cwc2 complex. The activated complex in turn binds to the promoters of blue lightregulated genes to repress the expression of filamentation genes. However, it is unclear at the moment whether the activated complex directly represses the filamentation or indirectly represses the process via the action of a downstream repressor. In model 1, the activated Cwc1/Cwc2 complex directly binds to the promoters of filamentation genes and negatively regulates the filamentation process. In model 2, an intermediate repressor is transcriptionally activated by the light-induced Cwc1/Cwc2 complex and then inhibits the production of dikaryotic filaments associated with the mating process. To reveal the real situation, a subtractive screen approach is currently underway to hopefully identify genes under direct control of Cwc1/ Cwc2 and also involved in light-regulated filamentation or additional biological processes in $C$. neoformans.

\section{Experimental procedures}

\section{Strains, media and growth conditions}

All $C$. neoformans strains used in this study are listed in Table 1, including two congenic serotype D MATa and MAT $\alpha$ strains JEC20 and JEC21, their auxotrophic derivatives and serotype A strain H99 (Edman and Kwon-Chung, 1990; 
A

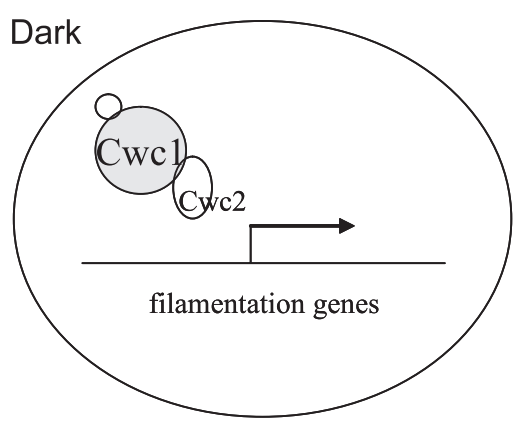

B

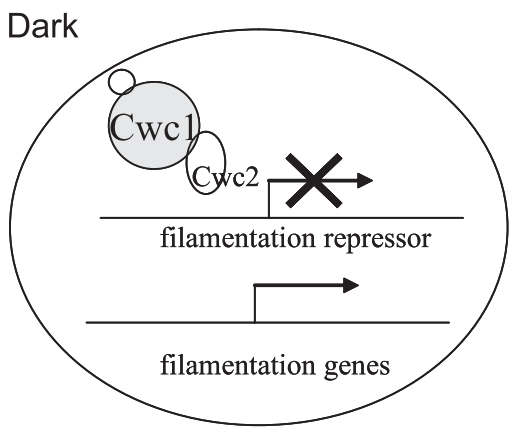

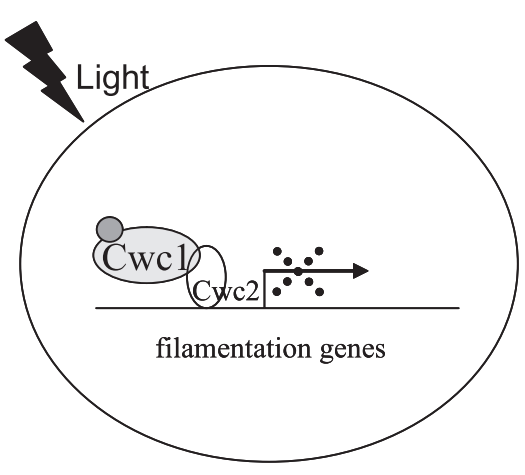

Fig. 6. Blue light negatively regulates the sexual filamentation via the Cwc1 and Cwc2 proteins in C. neoformans. Two models describing the blue light photoregulation in C. neoformans are proposed.

A. In model 1, the light-activated Cwc1/Cwc2 complex directly binds to the promoters and negatively regulates genes involved in the filamentation process.

B. In model 2, an intermediate repressor is transcriptionally activated by the light-activated Cwc1/Cwc2 complex and subsequently inhibits the production of dikaryotic filaments associated with the mating process.
Moore and Edman, 1993; Wang et al., 2000). All strains were handled according to standard techniques and media (Guthrie and Fink, 1991; Alspaugh et al., 1997). YPD medium, synthetic (SD) medium, V8 agar, SLAD medium and filament agar were prepared as described (Wickes et al., 1996; Alspaugh et al., 1997). C. neoformans strains were routinely maintained on YPD at $30^{\circ} \mathrm{C}$ and mating was conducted on V8 agar at $22^{\circ} \mathrm{C}$. Irradiation systems with different wavelengths were set up as previously described (Peters et al., 1998). Red light $\left(27 \mu \mathrm{mol} \mathrm{m} \mathrm{m}^{-2} \mathrm{~s}^{-1}\right)$ was generated from FL20SRF fluorescent tubes (National, Osaka, Japan) with a red, plastic filter (Shinkolite A No. 102, Mitsubishi Rayon, Tokyo, Japan). Far-red light $\left(33 \mu \mathrm{mol} \mathrm{m} \mathrm{m}^{-2} \mathrm{~s}^{-1}\right)$ was generated from FL20S-FR74 fluorescent tubes (Toshiba) wrapped with one layer of Polycolor No. 22 film and one layer of Polycolor No. 72 film (Tokyo Butai Shomei, Tokyo, Japan). Blue light $\left(11 \mu \mathrm{mol} \mathrm{m} \mathrm{m}^{-2} \mathrm{~s}^{-1}\right)$ was generated using FL20S.B fluorescent tubes (Toshiba). White light (84 or $7 \mu \mathrm{mol} \mathrm{m}^{-2} \mathrm{~s}^{-1}$ ) was generated from FL20BR fluorescent tubes (China Electric, Taipei, Taiwan).

Identification of the C. neoformans CWC1 and CWC2 homologues and molecular manipulation

The C. neoformans serotype D CWC1 and CWC2 gene was identified in the $C$. neoformans genome database with $N$. crassa WC-1 and WC-2. Two sets of PCR primers, WC1 (5'GGGCACGAATGGAGTGGTTTA-3') and WC4 (5'-GCGAT CAAGCCCTATTAAGGA-3') and WC272 (5'-GACCTTC CCTATACACACTGT-3') and WC284 (5'GCAGACACGTCTAGCTCTGTT-3'), were designed and used to amplify partial fragments of the $C$. neoformans CWC1 and CWC2 genes respectively. For CWC1, a 1583 bp PCR product of the expected size was subsequently used as a hybridization probe to identify the genomic clones from a JEC21 BAC library (Research Genetics). A $12 \mathrm{~kb} \mathrm{BamHI}$ genomic fragment was subcloned into pBluescript SK (+) (Stratagene) to generate a plasmid, PYKL1. The sequence of a 3790 bp region containing the $C$. neoformans CWC1

Table 1. Strains used in this study.

\begin{tabular}{|c|c|c|}
\hline Strain & Description & Reference \\
\hline JEC20 & MATa & $\begin{array}{l}\text { Kwon-Chung } \\
\text { et al. (1992) }\end{array}$ \\
\hline JEC21 & $M A T \alpha$ & $\begin{array}{l}\text { Kwon-Chung } \\
\text { et al. (1992) }\end{array}$ \\
\hline JEC34 & MATa ura5 & $\begin{array}{l}\text { Moore and } \\
\text { Edman (1993) }\end{array}$ \\
\hline JEC43 & MAT $\alpha$ ura5 & $\begin{array}{l}\text { Moore and } \\
\text { Edman (1993) }\end{array}$ \\
\hline H99 & $M A T \alpha$ & $\begin{array}{l}\text { Wang et al. } \\
(2000)\end{array}$ \\
\hline YKC7 & MAT $\alpha$ cwc1::URA5 ura5 & This Study \\
\hline YKC12 & MAT $\alpha$ cwc1::URA5 ura5 & This Study \\
\hline YKC29 & MATa cWc1::URA5 ura5 & This Study \\
\hline YKC25 & MAT $\alpha$ ura 5 cwc1 $\left(5-\mathrm{FOA}^{\top}\right)$ & This Study \\
\hline YKC30 & MATa ura5 cWc1 (5-FOA') & This Study \\
\hline YKC32 & MAT $\alpha$ ura5 cWc1 + CWC1-URA5 & This Study \\
\hline YKC33 & MAT $\alpha$ ura5 cWc1 + CWC1-URA5 & This Study \\
\hline YKC34 & MATa ura5 cWC1+CWC1-URA5 & This Study \\
\hline YKC38 & MAT $\alpha$ pGPD1::CWC1-URA5 ura5 & This Study \\
\hline YKC39 & MAT $\alpha$ pGPD1::CWC1-URA5 ura5 & This Study \\
\hline YKC40 & MAT $\alpha$ pGPD1::CWC1-URA5 ura5 & This Study \\
\hline KHC6 & MAT $\alpha$ GPD1::CWC2-URA5 ura5 & This Study \\
\hline
\end{tabular}


gene was verified with a series of designed sequencing primers. RT-PCR was performed and the amplified products were sequenced to verify the intron-exon junctions and determine a putative open reading frame. All nucleic acid manipulations were performed using standard procedures (Sambrook and Russell, 2001).

\section{Disruption, reintroduction and overexpression of the C. neoformans CWC1 gene}

To disrupt the $C$. neoformans CWC1 gene, a cWC1::URA5 disruption allele was constructed by replacing part of the CWC1 open reading frame with the URA5 marker. In brief, a Sall-mutated version of pBluescript SK (+) was first created and used to clone the $12 \mathrm{~kb} \mathrm{BamHI} \mathrm{CWC1} \mathrm{genomic} \mathrm{fragment}$ from $\mathrm{pYKL} 1$. The resulting plasmid $\mathrm{pYKL} 2$ was subsequently restriction digested with $X b a l$ and self-ligated to remove the $5^{\prime}$ part of $6 \mathrm{~kb}$ region to create pYKL3. pYKL3 was restriction digested with $X b a l$, blunt-ended by fill-in reaction, ligated with the Smal URA5 fragment released from plasmid pRCD69, and finally created PYKL4. The $4.5 \mathrm{~kb}$ fragment of the cwc1::URA5 disruption allele was released from pYKL4 by $X b a l$ and $E c o R I$ double digestion and introduced into the $M A T \alpha$ ura5 strain JEC43 by biolistic transformation as described (Toffaletti et al., 1993). One microgram of purified DNA was used for each bombardment in the transformation experiment. The resulting uracil prototrophic transformants were picked and screened by PCR and Southern blot analyses. To isolate MATa $c w c 1$ strains, the MAT $\alpha$ cwc1 strain was crossed with $C$. neoformans JEC34 strain (MATa ura5). The progeny were isolated on SD medium lacking uracil and the MATa strains containing the disruption construct were identified by PCR and Southern blot analyses.

The $C$. neoformans CWC1 reconstitution clone was made first by digesting pYKL2 with restriction enzyme Smal. The $11 \mathrm{~kb}$ vector containing fragment was gel-purified and selfligated to generate pYKL5. pYKL5 was linearized by BamHI and then ligated with the BamHI URA5 fragment released from plasmid pRCD69, and finally created reconstitution clone pYKL6. The ura5 version of the cwc1 MATa and MAT $\alpha$ mutant strains used for reconstitution were generated by selecting the original mutants on 5 -fluoroorotic acid (5-FOA) medium, which is toxic to URA5 cells. The CWC1 reconstitution plasmid pYKL6 was also biolistically transformed into the ura5 cWc1 MATa and MAT $\alpha$ mutant strains. Uracil prototrophic transformants were picked, screened by PCR and verified by Southern blot analysis.

To overexpress the CWC1 gene, the promoter region of $C$. neoformans GPD1 gene was amplified using PCR primers YK1 (5'-TATCCTATCTACAAAAATGCCGC TCTAGAAAC CAGTCA-3') and YK2 (5'-AAAAAGGATCCATGTATTTATG CAAGT ATACTCCTAGAAGGA-3'). The resulting $1.9 \mathrm{~kb}$ PCR product was purified, digested with $X b a l$ and $B a m H I$ and cloned into pBluescript SK $(+)$ (Stratagene) to generate pYKL7. The URA5 marker of pRCD69 was released by Xhol, filled-in, ligated with the Sacl digested and blunt-ended pBluescript SK (+) and generate pYKL8.

The $4.7 \mathrm{~kb}$ CWC1 fragment containing the coding region and the $0.5 \mathrm{~kb}$ downstream region was amplified by primers WC241 (5'-CGGGATCCATGTCAACA AACCTCACTTCT-3') and YK3 (5'-GTCATCAATAAGCTTGATGTGAGT-3'). The
PCR product was purified and digested with BamHI and ligated with PYKL8. The clone with correct orientation was verified and biolistically transformed into the MAT $\alpha$ ura5 strain JEC43. Uracil prototrophic transformants were picked and screened by PCR. The candidate clones were further verified by Northern blot and real-time PCR analyses.

\section{Overexpression of the C. neoformans CWC2 gene}

To overexpress the CWC2 gene, pYKL8 with the C. neoformans GPD1 promoter and URA5 selectable marker were also used. The $2 \mathrm{~kb}$ CWC2 fragment was amplified by primers WC325 (5'-CGGGATCCAATGTCC CTCCTCGCCGAGTCT-3') and WC326 (5'-TCCCCCGG GAAGGCCGAAATGAAATAAGCGT-3'). The PCR product was purified and digested with BamHI and Smal and ligated with pYKL8 to generate pKHS1. The clone was verified and biolistically transformed into the MAT $\alpha$ ura5 strain JEC43. Uracil prototrophic transformants were picked and screened by PCR. The candidate clones were further verified by realtime PCR analysis.

\section{Isolation of genomic DNA and Southern blot analysis}

Genomic DNA used for Southern analysis was prepared by a large-scale isolation protocol described previously (Perfect et al., 1993). DNA was digested with EcoRI and electrophoresed in a $0.8 \% 1 \times$ TAE agarose gel. DNA was transferred to nylon membrane (Immobilon ${ }^{\text {TM }}-\mathrm{Ny}+$, MILLIPORE) by capillary action. Southern hybridization was performed with a $0.8 \mathrm{~kb} W C 1$ probe generated by PCR amplification with the primers YK4 (5'-CCAGCTTGGACCATGGCTCCA GATCAG-3') and YK5 (5'-GGCGGGATATCGTAGTGGGT GCTGTAGAAGGG-3'). Probe was labelled by Prime- $\mathrm{It}^{\circledR} \|$ Random Primer Labeling Kit (Stratagene) with [ $\left.\alpha-{ }^{32} \mathrm{P}\right]-\mathrm{dCTP}$ (NEN Life Science Products) according to the manufacture's instruction.

\section{Gene expression analysis}

Strains subjected to gene expression analysis were first grown on YPD at $30^{\circ} \mathrm{C}$ for 2 days and then inoculated into YPD liquid medium. Cultures were shaken at 150 r.p.m. at $30^{\circ} \mathrm{C}$ with different illumination conditions for $22 \mathrm{~h}$. Illumination conditions included constant white light, constant darkness, or constant darkness with white light irradiation for $20 \mathrm{~min}$ or $2 \mathrm{~h}$ right before harvesting the cells. The CWC1 and CWC2 overexpression strains were also followed the same procedure and kept in darkness.

Cells were quickly harvested at the end of incubation, frozen in liquid nitrogen and freeze-dried. Total RNA was isolated from the freeze-dried cells using the TRIzol total RNA isolation reagent according to manufacturer's instructions (Invitrogene). For Northern blot analysis, $20 \mu \mathrm{g}$ of total RNA from each sample was separated by electrophoresis in a $1.2 \%$ agarose-formaldehyde gel. RNA was transferred by capillary action to nylon membrane (Immobilon ${ }^{\mathrm{TM}}-\mathrm{Ny}+$, MILLIPORE) and hybridized in hybridization buffer $[0.12 \mathrm{M}$ $\mathrm{Na}_{2} \mathrm{HPO}_{4}$ (pH 7.2), $0.25 \mathrm{M} \mathrm{NaCl}, 1 \mathrm{mM}$ EDTA (pH 8), $7 \%$ SDS and $50 \%$ formamide]. The same $0.8 \mathrm{~kb}$ CWC1 probe 
described in Southern analysis was also used in Northern hybridization analysis.

Real-time PCR analysis was performed using ABI 7000 Sequence Detection System (PE Applied-Biosystems) in the presence of SYBR-green. The optimization of the real-time PCR reaction was performed according to the manufacturer's instructions but scaled down to $25 \mu \mathrm{l}$ per reaction. The PCR was conducted with the SYBR ${ }^{\circledR}$ Green PCR Master Mix (PE Applied-Biosystems, 4309155). After optimization, $100 \mathrm{nM}$ PCR primers were used for the detection and quantification. PCR primers WC329 (5'-TATCCTTCGTTCGCCC-3') and WC330 (5'-ATCGGTGTTAGCACAG-3'), WC318 (5'-CGAC AGATACTCCACCG-3') and WC319 (5'-AGGACCCTTTC TCCATT-3'), and WC316 (5'-TTCTGAGAGCCCTGAGT-3') and WC317 (5'-GGCATCAACAC CAGCA-3') were used for the $C$. neoformans CWC1, CWC2 and GPD1 genes respectively.

\section{Mating assay}

Strains for mating and haploid fruiting assays were first grown on YPD at $30^{\circ} \mathrm{C}$ for 2 days. Mating reactions were conducted by co-incubating cells with desired partners on V8, SLAD or filament agar medium at $22^{\circ} \mathrm{C}$ in the light or dark for 15 days. The mating testers JEC20 (MATa) and JEC21 $(M A T \alpha)$ were used for serotype $D$ strains and serotype $A$ strain H99 was crossed with JEC20. Dikaryotic filaments at the periphery of the mating spot were evaluated under microscope. Pictures were taken 1-4 days post incubation.

\section{Nucleotide sequence accession number}

The sequence of the $C$. neoformans CWC1 gene has been submitted to GenBank and its accession number is AY660969.

\section{Acknowledgements}

We thank Dr Hsu-Liang Hsieh for kindly providing the facility with different irradiation systems. We thank Cristl Arndt and Dr Deborah S. Fox for technical assistance. We also thank Drs Shin-Hung Yeh, Janine Lin and Ya-Fen Chen for comments on the manuscript. We finally appreciate the sequence information provided by the $C$. neoformans Genome Project at Stanford Genome Technology Center. This work is supported by National Science Council Grants NSC93-2311-B002-032 to Dr Wei-Chiang Shen.

\section{References}

Alspaugh, J.A., Perfect, J.R., and Heitman, J. (1997) Cryptococcus neoformans mating and virulence are regulated by the G-protein $\alpha$ subunit GPA1 and cAMP. Genes Dev 11: 3206-3217.

Alspaugh, J.A., Davidson, R.C., and Heitman, J. (2000) Morphogenesis of Cryptococcus neoformans. In Dimorphism in Human Pathogenic and Apathogenic Yeasts, Vol. 5. Ernst, J.F., and Schmidt, A. (eds). Contributions to Microbiology. Basel: Karger, pp. 217-238.
Arpaia, G., Loros, J.J., Dunlap, J.C., Morelli, G., and Macino, G. (1993) The interplay of light and circadian clock. Independent dual regulation of clock-controlled gene ccg-2 (eas). Plant Physiol 102: 1299-1305.

Arpaia, G., Loros, J.J., Dunlap, J.C., Morelli, G., and Macino, G. (1995) Light induction of the clock-controlled gene ccg1 is not transduced through the circadian clock in Neurospora crassa. Mol Gen Genet 247: 157-163.

Ballario, P., Vittorioso, P., Magrelli, A., Talora, C., Cabibbo, A., and Macino, G. (1996) White collar-1, a central regulator of blue light responses in Neurospora, is a zinc finger protein. EMBO J 15: 1650-1657.

Ballario, P., Talora, C., Galli, D., Linden, H., and Macino, G. (1998) Roles in dimerization and blue light photoresponse of the PAS and LOV domains of Neurospora crassa white collar proteins. Mol Microbiol 29: 719-729.

Carattoli, A., Kato, E., Rodriguez-Franco, M., Stuart, W.D., and Macino, G. (1995) A chimeric light-regulated amino acid transport system allows the isolation of blue light regulator (b/r) mutants of Neurospora crassa. Proc Natl Acad Sci USA 92: 6612-6616.

Carlile, M.J. (1965) The photobiology of fungi. Annu Rev Plant Physiol 16: 175-202.

Casadevall, A., and Perfect, J.R. (1998) Cryptococcus neoformans. Washington, DC: American Society for Microbiology Press.

Cheng, P., Yang, Y., and Liu, Y. (2001) Interlocked feedback loops contribute to the robustness of the Neurospora circadian clock. Proc Natl Acad Sci USA 98: 7408-7413.

Cheng, P., Yang, Y., Gardner, K.H., and Liu, Y. (2002) PAS domain-mediated WC-1/WC-2 interaction is essential for maintaining the steady-state level of WC-1 and the function of both proteins in circadian clock and light responses of Neurospora. Mol Cell Biol 22: 517-524.

Cheng, P., Yang, Y., Wang, L., He, Q., and Liu, Y. (2003) WHITE COLLAR 1, a multifunctional Neurospora protein involved in the circadian feedback loops, light sensing, and transcription repression of wc-2. J Biol Chem 278: 38013808.

Christie, J.M., Salomon, M., Nozue, K., Wada, M., and Briggs, W.R. (1999) LOV (light, oxygen or voltage) domains of the blue-light photoreceptor phototropin (nph1): binding sites for the chromophore flavin mononucleotide. Proc Natl Acad Sci USA 96: 8779-8783.

Crosson, S., and Moffat, K. (2001) Structure of a flavinbinding plant photoreceptor domain: insights into lightmediated signal transduction. Proc Natl Acad Sci USA 98: 2995-3000.

Crosson, S., Rajagopal, S., and Moffat, K. (2003) The LOV domain family: photoresponsive signaling modules coupled to diverse output domains. Biochemistry 42: 2-10.

Degli-Innocenti, F., and Russo, V.E.A. (1984) Isolation of new white collar mutants of Neurospora crassa and studies on their behavior in the blue light-induced formation of protoperithecia. J Bacteriol 159: 757-761.

Dunlap, J.C. (1996) Genetic and molecular analysis of circadian rhythms. Annu Rev Genet 30: 579-601.

Edman, J.C., and Kwon-Chung, K.J. (1990) Isolation of the URA5 gene from Cryptococcus neoformans var. neoformans and its use as a selective marker for transformation. Mol Cell Biol 10: 4538-4544. 
Froehlich, A.C., Liu, Y., Loros, J.J., and Dunlap, J.C. (2002) White Collar-1, a circadian blue light photoreceptor, binding to the frequency promoter. Science 297: 815-819.

Griffith, G.W., Jenkins, G.I., Milner-White, E.J., and Clutterbuck, A.J. (1994) Homology at the amino acid level between plant phytochromes and a regulator of asexual sporulation in Emericella (=Aspergillus) nidulans. J Photochem Photobiol 59: 252-256.

Guthrie, C., and Fink, G.R. (1991) Guide to Yeast Genetics and Molecular Biology. San Diego: Academic Press.

Harding, R.W., and Melles, S. (1983) Genetic analysis of phototropism of Neurospora crassa perithecial beaks using white collar and albino mutants. Plant Physiol 72: 9961000.

Harding, R.W., and Turner, R.V. (1981) Photoregulation of the carotenoid biosynthetic pathway in albino and white collar mutants of Neurospora crassa. Plant Physiol 68: 745-749.

He, Q., Cheng, P., Yang, Y., Wang, L., Gardner, K.H., and Liu, Y. (2002) White collar-1, a DNA binding transcription factor and a light sensor. Science 297: 840-843.

Huala, E., Oeller, P.W., Liscum, E., Han, I.-S., Larsen, E., and Briggs, W.R. (1997) Arabidopsis NPH1: a protein kinase with a putative redox-sensing domain. Science 278: 2120-2123.

Hull, C.M., and Heitman, J. (2002) Genetics of Cryptococcus neoformans. Annu Rev Genet 36: 557-615.

Kwon-Chung, K.J. (1975) A new genus, Filobasidiella, the perfect state of Cryptococcus neoformans. Mycologia 67: 1197-1200.

Kwon-Chung, K.J. (1976) Morphogenesis of Filobasidiella neoformans, the sexual state of Cryptococcus neoformans. Mycologia 68: 821-833.

Kwon-Chung, K.J., Edman, J.C., and Wickes, B.L. (1992) Genetic association of making types and virulence in Cryptococcus neoformans. Infect Immun 60: 602-605.

Lauter, F.R., and Russo, V.E.A. (1991) Blue light induction of conidiation-specific genes in Neurospora crassa. Nucleic Acids Res 19: 6883-6886.

Lauter, F.R., Yamashiro, C.T., and Yanofsky, C. (1997) Light stimulation of conidiation in Neurospora crassa: studies with the wild-type strain and mutants $w c-1, w c-2$ and acon2. J Photochem Photobiol 37: 203-211.

Lengeler, K.B., Davidson, R.C., D'souza, C., Harashima, T., Shen, W.-C., Wang, P., et al. (2000) Signal transduction cascades regulating fungal development and virulence. Microbiol Mol Biol Rev 64: 746-785.

Linden, H., and Macino, G. (1997) White collar 2, a partner in blue light signal transduction, controlling expression of light-regulated genes in Neurospora crassa. EMBO J 16: 98-109.

Linden, H., Ballario, P., and Macino, G. (1997) Blue light regulation in Neurospora crassa. Fungal Genet Biol 22: 141-150.

Liu, Y., He, Q., and Cheng, P. (2003) Photoreception in Neurospora: a tale of two White Collar proteins. Cell Mol Life Sci 60: 2131-2138.

Mooney, J.L., and Yager, L.N. (1990) Light is required for conidiation in Aspergillus nidulans. Genes Dev 4: 14731482.

Moore, T.D.E., and Edman, J.C. (1993) The $\alpha$-mating type locus of Cryptococcus neoformans contains a peptide pheromone gene. Mol Cell Biol 13: 1962-1970.

Nelson, M.A., Morelli, G., Carattoli, A., Romano, N., and Macino, G. (1989) Molecular cloning of a Neurospora crassa carotenoid biosynthetic gene (albino-3) regulated by blue light and the products of the white collar genes. Mol Cell Biol 9: 1271-1276.

Perfect, J.R., Toffaletti, D.L., and Rude, T.H. (1993) The gene encoding phosphoribosylaminoimidazole carboxylase (ADE2) is essential for growth of Cryptococcus neoformans in cerebrospinal fluid. Infect Immun 61: 4446-4451.

Peters, J.L., SzError! Bookmark not defined.II, M., and Kendrick, R.E. (1998) The expression of light-regulated genes in the high-pigment-1 mutant of tomato. Plant Physiol 117: 797-807.

Sambrook, J., and Russell, D.W. (2001) Molecular Cloning: A Laboratory Manual, 3rd edn. Cold Spring Harbor, NY: Cold Spring Harbor Laboratory Press.

Sargent, M.L., and Briggs, W.R. (1967) The effects of light on a circadian rhythm of conidiation in Neurospora. Plant Physiol 42: 1504-1510.

Schmidhauser, T.J., Lauter, F.R., Russo, V.E.A., and Yanofsky, C. (1990) Cloning, sequence, and photoregulation of al-1, a carotenoid biosynthetic gene of Neurospora crassa. Mol Cell Biol 10: 5064-5070.

Schwerdtfeger, C., and Linden, H. (2000) Localization and light-dependent phosphorylation of white collar 1 and 2 , the two central components of blue light signaling in $\mathrm{Neu}$ rospora crassa. Eur J Biochem 267: 414-421.

Shen, W.-C., Davidson, R.C., Cox, G.M., and Heitman, J. (2002) Pheromones stimulate mating and differentiation via paracrine and autocrine signaling in Cryptococcus neoformans. Eukaryotic Cell 1: 366-377.

Sia, R.A., Lengeler, K.B., and Heitman, J. (2000) Diploid strains of the pathogenic basidiomycete Cryptococcus neoformans are thermally dimorphic. Fungal Genet Biol 29: $153-163$.

Tan, K.K. (1978) Light-induced fungal development. In The Filamentous Fungi, Vol. 3. Smith, J.E. and Berry, D.R. (eds). London: Edward Arnold, pp. 334-357.

Toffaletti, D.L., Rude, T.H., Johnston, S.A., Durack, D.T., and Perfect, J.R. (1993) Gene transfer in Cryptococcus neoformans by use of biolistic delivery of DNA. $J$ Bacteriol 175: 1405-1411.

Tscharke, R.L., Lazera, M., Chang, Y.C., Wickes, B.L., and Kwon-Chung, K.J. (2003) Haploid fruiting in Cryptococcus neoformans is not mating type $\alpha$-specific. Fungal Genet Biol 39: 230-237.

Wang, P., Perfect, J.R., and Heitman, J. (2000) The G-protein $\beta$ subunit GPB1 is required for mating and haploid fruiting in Cryptococcus neoformans. Mol Cell Biol 20: 352-362.

Wickes, B.L., Mayorga, M.E., Edman, U., and Edman, J.C. (1996) Dimorphism and haploid fruiting in Cryptococcus neoformans: association with the $\alpha$-mating type. Proc Natl Acad Sci USA 93: 7327-7331. 